\title{
Synthesis of ZnO Nanostructure by RTCVD Technique
}

\author{
Durgesh Wadhwa
}

\begin{abstract}
RTCVD technique are employed for production of several types of ZnO Nano-structure. Clusters of Nano-wires \& nanocrystals are aligned towards the nanostructures. The application of this types of nanowires are primarily employed in the LED's, optical equipment \& sensors etc. The outcomes of the $X R D$ discloses the wurtzite hexagonal stage of $\mathrm{ZnO}$. The specimens which are analyzed are authenticates the existent of impurities \& oxygen deficiency present in the samples.
\end{abstract}

Keywords: RTCVD, Nano-structure, nanowires, nanocrystals, $\mathrm{ZnO}$, wurtzite hexagonal phase

\section{INTRODUCTION}

The nanostructure of the Zinc Oxides are produced by the various procedures, according their applications. There are various applications of the $\mathrm{ZnO}$ nanocrystals, some of them are sensing devices (gas, chemical \&biosensors), optoelectronics and light emitting devices, transparent/apparent conductors in solar cells, in addition to in high-power electrical \& electronics [1]-[3].

The Zinc oxide nanostructure are also applicable in the Ultraviolet UV device, photodetectors, piezo-transducers, hydrogen storage, together with spintronic devices in consequence of its optical \& electrical properties. Different procedures for the generation of $\mathrm{ZnO}$ Nano-structures are discussed \& published in various journals such as solution technique, sputtering, hydrothermal procedure, thermal evaporation, these techniques for synthesis are analyzed either for their characteristics or for their application in various fields [4]-[6].

These materials/resources are marketable as a result of their high band gap for the optoelectronic equipment. The $\mathrm{ZnO}$ is crucial for various applications as a result of its prime characteristics like great exciton binding power/energy $(60 \mathrm{meV}) \&$ the high optical gain of $300 \mathrm{~cm}^{-1}$ [7].

As described many journals are already published/printed on this, therefore, this paper represent the analyzed results of the morphological variation \& structural features of the Zink Oxide capable for the assistance in manufacturing of the various products and equipment like LEDs, products expressing the features of the optoelectronics [8], [9].

\section{DETAILS OF THE EXPERIMENT}

Different techniques are accessible for synthesis of the zinc oxide. Herein, this experiment employing the thermal chemical vapor deposition (RTCVD). In this method pure zinc is vaporized in the presence of surplus oxygen. The little evaporation compartment generate of quartz tubes are amended into thermal evaporation system for the assembly

Revised Manuscript Received on August 05, 2019.

Dr. Durgesh Wadhwa, Department of Chemistry, Sanskriti University, UttarPradesh, India.(E-mail: sanpubip@gmail.com) of the RTCVD setup. The evaporation compartment is open for evaporation of specimen. For the evaporation source, gas inlet, specimen holder, gas outlet as demonstrated in Figure 1. One of the tough job of this experiment is to contain the vaporized material \& to uphold the uniform $\mathrm{O}_{2}$ pressure in the compartment, quartz tube helps to execute this job [10], [11].

The $\mathrm{Zn}$ powder is encapsulated (contained by the Moboat) in the quartz tube comportment $\&$ the entire assembly is encircled in the vacuum chamber. The supply of oxygen is need throughout the experiment, so that the compartment was move/clear out form $10^{-5}$ to .1 TORR \& oxygen is injected into the sub compartment after loading the substrates \& the source materials. The variation in structures $\&$ the morphologies of the specimen is caused by the variation of the source temperature from $600^{\circ} \mathrm{C}$ to $700^{\circ} \mathrm{C}$ [12].

The fundamental \& structural characteristics of the specimen was analyzed by the deposits on the quartz tube. The preparation of the specimens are executed individually by maintaining a continuous temperature at 0.1 TORR oxygen pressure.

"The Bragg angle is calculated by the X-ray diffraction measurement, which is performed by employing the Bruker $D 8$-advance diffractometer with $\mathrm{CuK \alpha}$ radiation $(\lambda=1.542$ $\bar{A})$. JEOL (JSM-6380) electron microscope was employed for the obtaining the Scanning Electron Microscopic (SEM) Images. By employing the LabRam HR800 JY with the HeNe source $(\lambda=632.8 \mathrm{~nm})$, the Ram spectra was obtained \& beam diameter of $1.2 \mu \mathrm{m}$ operating at fixed laser power of 2 $m W$ and scanning range of 200-650 $\mathrm{cm}^{-1, " . ~[13], ~[14] ~}$

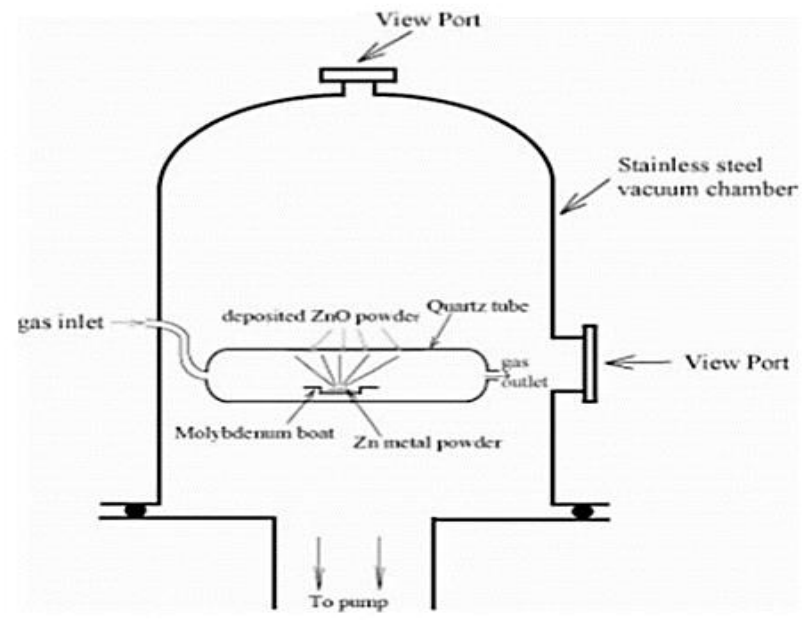

Figure 1. Experimental/trail set up of RTCVD system used for the growth/development of $\mathrm{ZnO}$ nanostructures

Blue Eyes Intelligence Engineering

\& Sciences Publication 


\section{RESULTS \& DISCUSSION}

The diffraction pattern of the X-ray of $\mathrm{ZnO}$ nanostructures are illustrated as a purpose of deposition temperature $\left(600^{\circ}\right.$ to $\left.700^{\circ} \mathrm{C}\right)$ in Figure 2 . The temperature is observed for the variation in the peak intensities. The peak intensities, which are observed are matched with standard peaks of wurtzite hexagonal phase related to the JCPDS files. The JCPDS files are preferably 36-1451, 21-1486, \& 01-1136.

The best crystal grown are represented by the intense peaks. The peak strength connected to (002) plane raised with growing deposition temperature demonstrating that coordination is without airs by the growing temperature, though the intensity of additional peaks connected to (100), $\&$ (002), \& (101), \& (102), \& (110), \& (103), \& (112), \& (201), \& (004) \& (202) has altered. The peak associated with (110), \& (002), \& (101), \& (102), \& (110), \& (103), \& (200), \& (112) \& (002) displays low intensity.

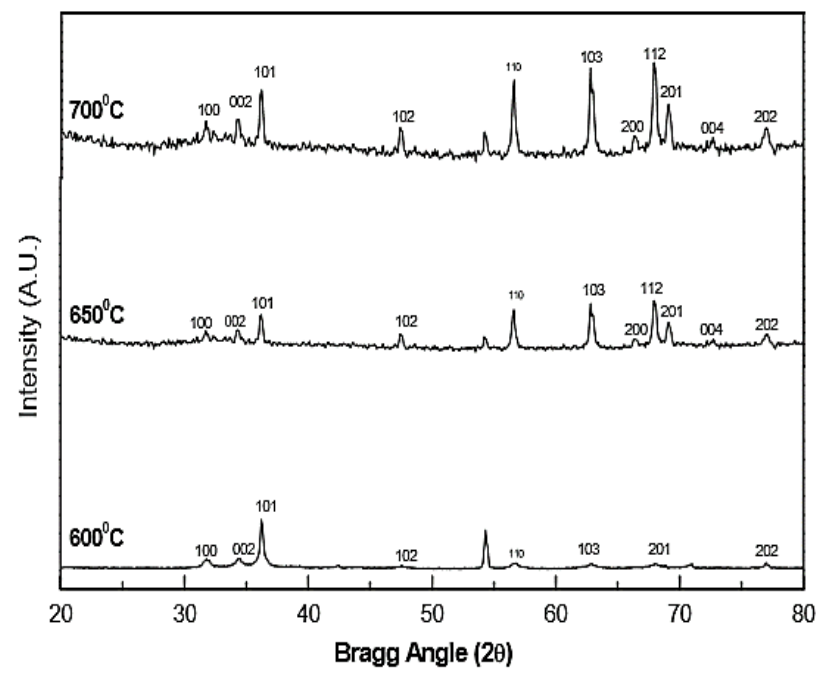

Figure 2. X-ray diffraction/scattering configurations of $\mathrm{ZnO}$ nanostructures
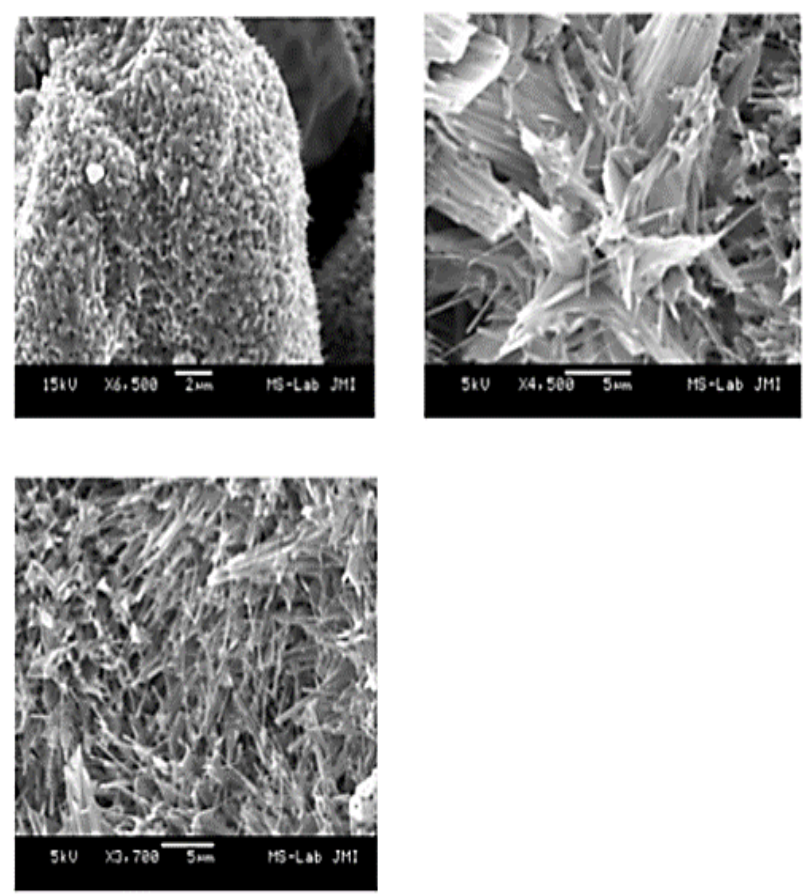

Figure 3. SEM Images of $\mathrm{ZnO}$
The SEM image of the Zink oxide nanostructure is illustrated in the Figure 3. As a function of deposition temperature. Very tiny nanocrystals of zinc oxide are created with a width/diameter of 90-150 nm. Formation of bundles of nanowires (diameter ranging 10-20 nm) are created, when temperature of the compartment elevated to $650^{\circ} \mathrm{C}$. Nanowire bundles are organized vertically and at $700^{\circ} \mathrm{C}$ these nanowires are separated, which is observed in SEM image.

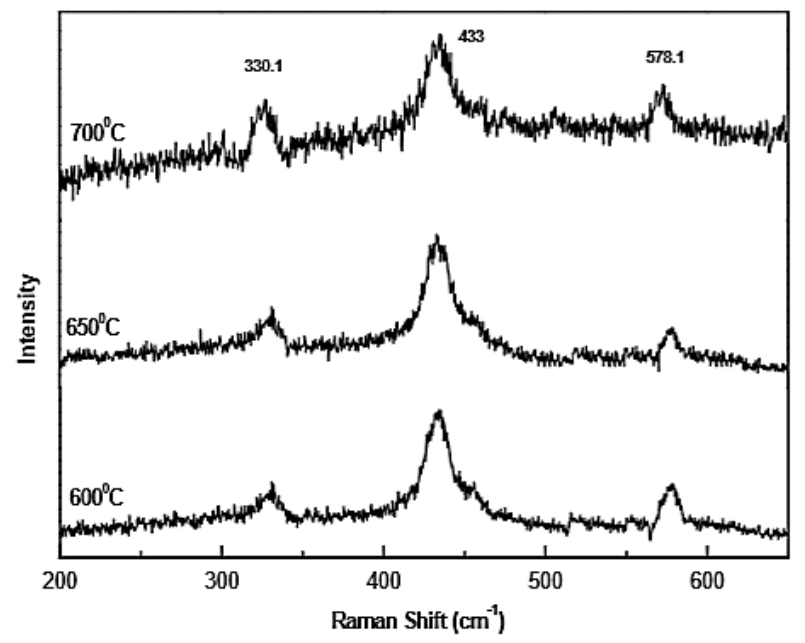

Figure 4. Raman spectra of nanostructures

To determine morphology \& nanostructure's size the Raman spectroscopy was performed because of its sensitivity towards it. The Raman spectra displays 3 different peaks surrounding to $330 \mathrm{~cm}^{-1}, 434 \mathrm{~cm}^{-1} \& 578$ $\mathrm{cm}^{-1}$, these spectra acclaimed the occurrence of Wurtzite $\mathrm{ZnO}$ phase as displayed in Figure 4 according to the structure/deposition the peak location may slightly differ for every specimen. Herein, 3 various types of peaks are detected:

A peak around 326.5 to $330.0 \mathrm{~cm}^{-1}$, which is suppressed \& broad \& belongs to lower E2 \& correlated to several photons scattering process.

$>\quad$ A peak about 433-435.q $\mathrm{cm}^{-1}$, which is E2 higher peak attached to the $\mathrm{O}_{2}$ atoms are the most dominant phonon peaks.

$>\quad$ A peak about 572.5 to $578 \mathrm{~cm}^{-1}$ that is of E1 (LO) mode, which is relatively symmetric peak.

The E1 (LO) peak represents the impurities, $\mathrm{O}_{2}$ short locations and $\mathrm{Zn}$ interstitial site.

\section{CONCLUSION}

In this research, numerous $\mathrm{ZnO}$ nanostructures are grown like as nanowires, \& nanocrystals are in form of bunches, these growth are done by varying the temperature of the growth at constant $\mathrm{O}_{2}$ pressure of 0.100 torr in the compartment during the rapid thermal evaporation. XRD, SEM \& the Raman were employed to characterize the grown nanostructures. The peaks formed during the X-Ray test are used to govern the impurities, oxygen deficient sites and $\mathrm{Zn}$ interstitial sites exists in the sample.

Published By:

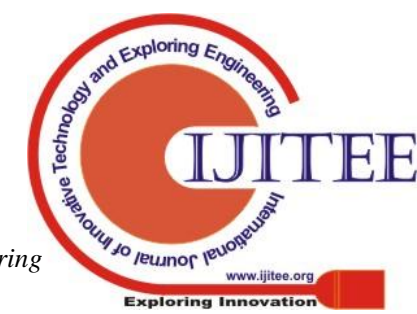




\section{REFERENCE}

1. J. Xing, Z. Wang, C. Sun, and M. Ji, "Synthesis and Characterization of a Novel Near-Infrared Fluorescent Probe for Living Cells Imaging," J. Nanosci. Nanotechnol., vol. 20, no. 2, pp. 668-672, Feb. 2020.

2. A. A. Dudhane, S. R. Waghmode, L. B. Dama, V. P. Mhaindarkar, A. Sonawane, and S. Katariya, "Synthesis and Characterization of Gold Nanoparticles using Plant Extract of Terminalia arjuna with Antibacterial Activity," Int. J. Nanosci. Nanotechnol., vol. 15, no. 2, pp. 75-82, May 2019.

3. G. Cheraghian, S. S. Khalili Nezhad, and S. Bazgir, "Improvement of Thermal Stability of Polyacryl amide Solution Used as a Nano-fluid in Enhanced Oil Recovery process by Nanoclay," Int. J. Nanosci. Nanotechnol., vol. 11, no. 3, pp. 201-208, Sep. 2015.

4. N. El-Atab et al., "Diode behavior in ultra-thin low temperature ALD grown zinc-oxide on silicon," AIP $A d v$. , vol. 3, no. 10, p. 102119, Oct. 2013.

5. Y. T. Shih, C. Y. Chiu, C. W. Chang, J. R. Yang, M. Shiojiri, and M. J. Chen, "Stimulated Emission in Highly (0001)-Oriented $\mathrm{ZnO}$ Films Grown by Atomic Layer Deposition on the Amorphous Glass Substrates," J. Electrochem. Soc., vol. 157, no. 9, p. H879, Sep. 2010.

6. M.-J. Chen, J.-R. Yang, and M. Shiojiri, "ZnO-based ultra-violet light emitting diodes and nanostructures fabricated by atomic layer deposition," Semicond. Sci. Technol., vol. 27, no. 7, p. 074005, Jul. 2012.

7. W. C. LI et al., "STRUCTURAL INVESTIGATION OF n- ZnO /p- GaN ULTRAVIOLET LIGHT-EMITTING DIODES GROWN BY ATOMIC LAYER DEPOSITION," Funct. Mater. Lett., vol. 04, no. 03, pp. 221-224, Sep. 2011.

8. Ü. Özgür et al., "A comprehensive review of $\mathrm{ZnO}$ materials and devices," J. Appl. Phys., vol. 98, no. 4, p. 041301, Aug. 2005.

9. J.-T. Chen et al., "Catalytic growth of semiconducting zinc oxide nanowires and their photoluminescence properties," 1998.

10. Yousheng Zhang, Lisheng Wang, Xiaohua Liu, Yunjie Yan, and Changqiang Chen, and J. Zhu*, "Synthesis of Nano/Micro Zinc Oxide Rods and Arrays by Thermal Evaporation Approach on Cylindrical Shape Substrate," 2005.

11. M. Ahmad and J. Zhu, " $\mathrm{ZnO}$ based advanced functional nanostructures: synthesis, properties and applications," $J$. Mater. Chem., vol. 21, no. 3, pp. 599-614, Dec. 2011.

12. †, $\dagger$ Jr H. He, $\uparrow$ Chang S. Lao, * $\dagger$ Lih J. Chen, $\S$ and Dragomir Davidovic, and $\dagger$ Zhong L. Wang*, "LargeScale Ni-Doped ZnO Nanowire Arrays and Electrical and Optical Properties," 2005.

13. X. Chen et al., " $\mathrm{ZnO}$ nanorod/GaN light-emitting diodes: The origin of yellow and violet emission bands under reverse and forward bias," J. Appl. Phys., vol. 110, no. 9, p. 094513, Nov. 2011.

14. M. Ali and M. Winterer, "ZnO Nanocrystals: Surprisingly 'Alive,"” Chem. Mater., vol. 22, no. 1, pp. 85-91, Jan. 2010. 